\title{
DECOUPLING INEQUALITIES AND SOME MEAN-VALUE THEOREMS
}

\author{
JEAN BOURGAIN
}

\begin{abstract}
The purpose of this paper is to present some further applications of the general decoupling theory from [B-D1, 2] to certain diophantine issues. In particular, we consider mean value estimates relevant to the Bombieri-Iwaniec approach to exponential sums and arising in the work of Robert and Sargos $[\mathrm{R}-\mathrm{S}]$. Our main input is a new mean value theorem.
\end{abstract}

\section{Summary}

The aim of this Note is to illustrate how a version of the general decoupling inequality for hypersurfaces established in [B-D permits to recover certain known mean-value theorems in number theory and establish some new ones. Easy applications in this direction were already pointed out in [B-D] and the material presented here is a further development. Our main emphasis will be on the method rather than the best exponents that can be obtained this way.

In the first section, we state a form of the main decoupling theorem from B-D] to the situation of smooth hyper surfaces in $\mathbb{R}^{n}$ with non-degenerate (but not necessarily definite) second fundamental form (a detailed argument appears in [B-D2]). The motivation for this appears in Sections 2 and 3, which aims at proving decoupling inequalities for real analytic curves $\Gamma \subset \mathbb{R}^{n}$ not contained in a hyperplane. The assumption of real analyticity is purely for convenience (it suffices for the subsequent applications) and a similar result also holds in the smooth category. An $(n-1)$-fold convolution of $\Gamma$ leads indeed to a hypersurface $S \subset \mathbb{R}^{n}$ of non-vanishing curvature. The relevant statement is inequality (3.2) below with moment $q=2(n+1)$, where we consider the multi-linear (i.e. $(n-1)$-linear) setting. The next step is to reformulate this inequality as a mean-value theorem for exponential sums stated as Theorem 1, which is a quite general and optimal result. Our first focus point are certain mean value inequalities arising in the Bombieri-Iwaniec 
approach [B-I1, 2] to exponential sums and the subsequent developments of this technique (see $[\mathrm{H}]$ for the complete exposition). More specifically, Theorem 1 is relevant to the so-called 'first spacing problem' which is analytically captured by mean-value expressions of the type

$$
\begin{gathered}
N_{8}(\delta)=\int_{0}^{1} \int_{0}^{1} \int_{0}^{1}\left|\sum_{n \sim N} e\left(x_{0} n+x_{1} n^{2}+x_{2} \frac{1}{\delta}\left(\frac{n}{N}\right)^{\frac{3}{2}}\right)\right|^{8} d x_{0} d x_{1} d x_{2} \\
N_{10}(\delta, N \delta)=\int_{0}^{1} \int_{0}^{1} \int_{0}^{1} \int_{0}^{1} \mid \sum_{n \sim N} e\left(x_{0} n+x_{1} n^{2}+x_{2} \frac{1}{\delta}\left(\frac{n}{N}\right)^{\frac{3}{2}}+\left.x_{3} \frac{1}{N \delta}\left(\frac{n}{N}\right)^{\frac{1}{2}}\right|^{10} d x_{0} d x_{1} d x_{2} d x_{3}\right.
\end{gathered}
$$

and

$$
N_{12}(\delta, N \delta)=\int_{0}^{1} \int_{0}^{1} \int_{0}^{1} \int_{0}^{1} \mid \sum_{n \sim N} e\left(x_{0} n+x_{1} n^{2}+x_{2} \frac{1}{\delta}\left(\frac{n}{N}\right)^{3 / 2}+\left.x_{3} \frac{1}{N \delta}\left(\frac{n}{N}\right)^{\frac{1}{2}}\right|^{12} d x_{0} d x_{1} d x_{2} d x_{3} .\right.
$$

In the application, the most important range of $\delta$ is $\delta \sim \frac{1}{N^{2}}$. As a special case of a more general result, it was proven in [B-I2] that

$$
N_{8}(\delta) \ll \delta N^{5+\varepsilon}+N^{4+\varepsilon}
$$

and in $[\mathrm{H}-\mathrm{K}]$ that

$$
N_{10}(\delta, N \delta) \ll \delta N^{7+\varepsilon}+N^{5+\varepsilon} .
$$

Our Fourier analytical approach gives a quite different treatment and unified approach to this problem. In particular, Theorem 9 in Section 4 below shows that in fact

$$
N_{10}(\delta, N \delta) \ll N^{5+\varepsilon} \text { for } \delta<N^{-\frac{33}{18}} .
$$

Since however the main contribution (at least in the treatment $[\mathrm{H}]$ ) in the exponential sum problem

$$
\sum_{m \sim M} e\left(T F\left(\frac{m}{M}\right)\right)
$$

has $\delta=\frac{1}{N^{2}}$, the improvement $(0.6)$ does not lead to new results on this matter.

Our next application are certain mean value results in the work of Robert and Sargos [R-S]. It is proven in $[\mathrm{R}-\mathrm{S}]$ that

$$
I_{6}\left(N^{-3}\right)=\int_{0}^{1} \int_{0}^{1}\left|\sum_{n \sim N} e\left(n^{2} x+N^{-3} n^{4} y\right)\right|^{6} d x d y \ll N^{3+\varepsilon}
$$




$$
\begin{gathered}
I_{8}\left(N^{-\frac{5}{2}}\right)=\int_{0}^{1} \int_{0}^{1}\left(\left.\sum_{n \sim N} e\left(n^{2} x+N^{-\frac{5}{2}} n^{4} y\right)\right|^{8} d x d y \ll N^{\frac{9}{2}+\varepsilon}\right. \\
I_{10}\left(N^{-\frac{17}{8}}\right)=\int_{0}^{1} \int_{0}^{1}\left|\sum_{n \sim N} e\left(n^{2} x+N^{-\frac{17}{8}} n^{4} y\right)\right|^{10} d x d y \ll N^{\frac{49}{8}+\varepsilon}
\end{gathered}
$$

Inequality (0.11) is the optimal statement for the 6th moment (a different proof using the decoupling theorem for curves appears in [B-D]). While (0.12), (0.13) are essentially sharp, they are not the optimal results for the 8th and 10th moment respectively. Since $I_{p}(\lambda)$ is a decreasing function of $\lambda$ for $p$ an even integer, (0.13) obviously implies that

$$
I_{10}=\int_{0}^{1} \int_{0}^{1}\left|\sum_{n \sim N} e\left(n^{2} x+n^{4} y\right)\right|^{10} d x d y \ll N^{\frac{49}{8}+\varepsilon} .
$$

In $[\mathrm{R}-\mathrm{S}]$ an application of (0.14) to Weyl's inequity is given, following a method initiated by Heath-Brown. In view of the present state of the art, the relevant statement is the bound

$$
\left|f_{8}(\alpha ; N)\right|=\left|\sum_{1 \leq n \leq N} e\left(\alpha n^{8}\right)\right| \ll N^{1-3.2^{-8}}\left(N^{4} q^{-1}+1+q N^{-4}\right)^{\frac{1}{160}}
$$

assuming $\left|\alpha-\frac{a}{q}\right| \leq q^{-2}, q \geq 1,(a, q)=1$ (though the exponent $\sigma(8)=$ $3.2^{-8}=0,01171 \cdots$ is superseded by a recent result of Wooley, see Theorem 7.3 in [W2, which gives in particular $\sigma(8)=\frac{1}{2.7 .6}=\frac{1}{84}=0,01190 \cdots$ ).

More recently, inequality $(0.14)$ has been improved in $[\mathrm{P}]$ to

$$
I_{10} \ll N^{6+\varepsilon}
$$

using a different more arithmetical approach. As a consequence the first factor in the r.h.s. of $(0.15)$ is replaced by $N^{1-\frac{16}{5} \cdot 2^{-8}}$, i.e. $\sigma(8)=\frac{1}{18}=$ $0,0125 \cdots$.

In the final section of this paper, we establish the bounds

$$
\begin{gathered}
I_{8}\left(N^{-\frac{7}{3}}\right) \ll N^{\frac{13}{3}+\varepsilon} \\
I_{10} \leq I_{10}\left(N^{-\frac{5}{3}}\right) \ll N^{\frac{17}{3}+\varepsilon}
\end{gathered}
$$

implying a corresponding improvement $\sigma(8)=\frac{56}{15} 2^{-8}=0,0145 \ldots$ in Weyl's inequality. 


\section{Decoupling inequality for SMOoth hypersurfaces With}

Let us start by recalling the main result from [B-D], which is the so-called $\ell^{2}$-decoupling theorem for the Fourier transform of distributions carried by hypersurfaces in $\mathbb{R}^{n}$ of positive curvature. This is a quite general harmonic analysis result with diverse applications, in particular to PDE's and spectral theory (see [B-D] for some of these).

In order to formulate the result, we need some terminology. Let $S \subset$ $\mathbb{R}^{n}$ be a compact smooth hypersurface of positive curvature and denote $S_{\delta}(\delta>0$ a small parameter $)$ a $\delta$-neighborhood of $S$. Decompose $S_{\delta}$ as a union of tangent $\underbrace{\sqrt{\delta} \times \cdots \times \sqrt{\delta}}_{n-1} \times \delta$ boxes $\tau$ with bounded overlap. Denoting $B_{R} \subset \mathbb{R}^{n}$ a ball of radius $R$, the following inequality holds for functions $f$ s.t. $\operatorname{supp} \hat{f} \subset S_{\delta}$

$$
\|f\|_{L^{p}\left(B_{\frac{1}{\delta}}\right)} \ll \delta^{-\varepsilon}\left(\sum_{\tau}\left\|f_{\tau}\right\|_{L^{p}\left(B_{\frac{1}{\delta}}\right)}^{2}\right)^{\frac{1}{2}} \text { with } p=\frac{2(n+1)}{n-1}
$$

and $f_{\tau}=\left(\left.\hat{f}\right|_{\tau}\right)^{\vee}$ denoting the Fourier restriction of $f$ to the tile $\tau$.

By interpolation, (1.1) of course also holds for $2 \leq p \leq \frac{2(n+1)}{n-1}$ while for $\frac{2(n+1)}{n-1} \leq p \leq \infty$, the inequality becomes

$$
\|f\|_{L^{p}\left(B_{\frac{1}{\delta}}\right)} \ll \delta^{-\frac{n-1}{4}+\frac{n+1}{2 p}-\varepsilon}\left(\sum_{\tau}\left\|f_{\tau}\right\|_{L^{p}\left(B_{\frac{1}{\delta}}\right)}^{2}\right)^{\frac{1}{2}} .
$$

Next, let us relax the assumption on $S$, requiring $S$ to have non-degenerate (but not necessarily definite) second fundamental form. A statement such as (1.1) can not be valid any more. For instance, if $S \subset \mathbb{R}^{3}$ is a ruled surface, we may take supp $\hat{f}$ in a $\sqrt{\delta}$-neighborhood of a straight line segment with only the obvious decoupling available. This problem of curvature break-down for lower dimensional sections of $S$ can be bypassed by a suitable reformulation of the decoupling property. Assuming $S$ as above and supp $\hat{f} \subset S_{\delta}$, one has for $\frac{2(n+1)}{n-1} \leq p \leq \infty$

$$
\|f\|_{L^{p}\left(B_{\frac{1}{\delta}}\right)} \ll \delta^{-\frac{n-1}{2}+\frac{n}{p}-\varepsilon}\left(\sum_{\tau}\left\|f_{\tau}\right\|_{L^{p}\left(B_{\frac{1}{\delta}}\right)}^{p}\right)^{\frac{1}{p}} .
$$


This statement is weaker than (1.2) but will perform equally well in what follows because in the applications below supp $\hat{f}$ will be uniformly spread out over $S$.

The proof of (1.3) requires a modification of the argument in [B-D] (for positive curvature). Details appear in B-D2]. Our next goal is to derive from (1.3) a decoupling inequality for curves $\Gamma \subset \mathbb{R}^{n}$ not lying in a hyperplane and which will imply our Theorem 1.

\section{Construction of hypersurfaces from Curves}

Let $\Gamma \subset \mathbb{R}^{n}$ be parametrized by $\Phi:[0,1] \rightarrow \mathbb{R}^{n}: t \rightarrow\left(t, \varphi_{1}(t), \ldots, \varphi_{n-1}(t)\right)$ where we assume for simplicity that $\varphi_{1}, \ldots, \varphi_{n-1}$ are real analytic and (importantly) that $1, t, \varphi_{1}, \ldots, \varphi_{n-1}$ linearly independent. In particular, $\Gamma$ does not lie in a hypersurface. Our assumption means non-vanishing of the Wronskian determinant

$$
W\left(\varphi_{1}^{\prime \prime}, \ldots, \varphi_{n-1}^{\prime \prime}\right) \neq 0
$$

We build a hypersurface $S \subset \mathbb{R}^{n}$ as $(n-1)$-fold sum set

$$
S=\Gamma_{1}+\cdots+\Gamma_{n-1}
$$

where $\Gamma_{j}=\Phi\left(I_{j}\right)$ and $I_{1}, \ldots, I_{n-1} \subset I \subset[0,1]$ are fixed consecutive disjoint subintervals. Hence $S$ is parametrized by

$$
\left\{\begin{array}{l}
x_{0}=t_{1}+\ldots t_{n-1} \\
x_{1}=\phi_{1}\left(t_{1}\right)+\cdots+\phi_{1}\left(t_{n-1}\right) \\
\vdots \\
x_{n-1}=\phi_{n-1}\left(t_{1}\right)+\cdots+\phi_{n-1}\left(t_{n-1}\right)
\end{array}\right.
$$

with $t_{j} \in I_{j}$. Our aim is to show that the second fundamental form of $S$ is non-degenerate (but note that it may be indefinite). 
Perturb $t=\left(t_{1}, \ldots, t_{n-1}\right) \in I_{1} \times \cdots \times I_{n-1}$ to $\left(t_{1}+s_{1}, \ldots, t_{n-1}+s_{n-1}\right)$, $\left|s_{j}\right|=o(1)$, obtaining

$$
\left\{\begin{array}{l}
x_{0}-t_{1}-\cdots-t_{n-1} \equiv x_{0}^{\prime}=s_{1}+\cdots+s_{n-1} \\
\left(\begin{array}{c}
x_{1}-\phi_{1}\left(t_{1}\right)-\cdots-\phi_{1}\left(t_{n-1}\right) \\
\vdots \\
x_{n-1}-\phi_{n-1}\left(t_{1}\right)-\cdots-\phi_{n-1}\left(t_{n-1}\right)
\end{array}\right) \equiv\left(\begin{array}{c}
x_{1}^{\prime} \\
\vdots \\
x_{n-1}^{\prime}
\end{array}\right)=D_{1}\left(\begin{array}{c}
s_{1} \\
\vdots \\
s_{n-1}
\end{array}\right)+\frac{1}{2} D_{2}\left(\begin{array}{c}
s_{1}^{2} \\
\vdots \\
s_{n-1}^{2}
\end{array}\right)+O\left(|s|^{3}\right)
\end{array}\right.
$$

with

$$
D_{1}=\left[\begin{array}{ccc}
\phi_{1}^{\prime}\left(t_{1}\right) & \cdots & \phi_{1}^{\prime}\left(t_{n-1}\right) \\
\vdots & \vdots \\
\phi_{n-1}^{\prime}\left(t_{1}\right) & \cdots & \phi_{n-1}^{\prime}\left(t_{n-1}\right)
\end{array}\right] \text { and } D_{2}=\left[\begin{array}{c}
\phi_{1}^{\prime \prime}\left(t_{1}\right) \cdots \phi_{1}^{\prime \prime}\left(t_{n-1}\right) \\
\vdots \\
\phi_{n-1}^{\prime \prime \prime}\left(t_{1}\right) \cdots \phi_{n-1}^{\prime \prime}\left(t_{n-1}\right)
\end{array}\right] \text {. }
$$

The non-vanishing of $\operatorname{det} D_{1}$ can be derived from the non-vanishing of $W\left(\phi_{1}^{\prime}, \ldots, \phi_{n-1}^{\prime}\right)$ which is a consequence of our assumption (2.1).

Hence, since $D_{1}$ is invertible and denoting $\xi=(1, \ldots, 1) \in \mathbb{R}^{n-1}$, the first equation in (2.4) gives

$$
\begin{aligned}
x_{0}^{\prime} & =\left\langle D_{1}^{-1}\left(\begin{array}{c}
x_{1}^{\prime} \\
\vdots \\
x_{n-1}^{\prime}
\end{array}\right), \xi\right\rangle-\frac{1}{2} \sum_{j=1}^{n-1} s_{j}^{2}\left\langle D_{1}^{-1} D_{2} e_{j}, \xi\right\rangle+O\left(|s|^{3}\right) \\
& =\left\langle\left(\begin{array}{c}
x_{1}^{\prime \prime} \\
\vdots \\
x_{n-1}^{\prime \prime}
\end{array}\right), \xi\right\rangle-\frac{1}{2} \sum_{j=1}^{n-1}\left(x_{j}^{\prime \prime}\right)^{2}\left\langle D_{1}^{-1} D_{2} e_{j}, \xi\right\rangle+O\left(\left|x^{\prime \prime}\right|^{3}\right)
\end{aligned}
$$

where

$$
\left(\begin{array}{c}
x_{1}^{\prime \prime} \\
\vdots \\
x_{n-1}^{\prime \prime}
\end{array}\right)=D_{1}^{-1}\left(\begin{array}{c}
x_{1}^{\prime} \\
\vdots \\
x_{n-1}^{\prime}
\end{array}\right)
$$

From (2.6), it remains to ensure that

$$
\left\langle D_{1}^{-1} D_{2} e_{j}, \xi\right\rangle \neq 0 \text { for each } j=1, \ldots, n-1 .
$$

Take $j=1$. Up to a multiplicative factor, 


$$
\left\langle D_{2} e_{1},\left(D_{1}^{-1}\right)^{*} \xi\right\rangle \doteq \sum_{k=1}^{n-1} \phi_{k}^{\prime \prime}\left(t_{1}\right)\left|\begin{array}{ccc}
\phi_{1}^{\prime}\left(t_{1}\right) & \cdots & \phi_{1}^{\prime}\left(t_{n-1}\right) \\
\vdots & & \\
1 & \cdots & 1 \\
\vdots & & \\
\phi_{n-1}^{\prime}\left(t_{1}\right) \cdots & \phi_{n-1}^{\prime}\left(t_{n-1}\right)
\end{array}\right|-k .
$$

By the mean-value theorem, we obtain separated $t_{1}<t_{2}^{\prime}<\cdots<t_{n-1}^{\prime}$ such that

$$
\begin{aligned}
(2.8) & =\sum_{k=1}^{n-1}(-1)^{k} \phi_{k}^{\prime \prime}\left(t_{1}\right)\left|\begin{array}{c}
\phi_{1}^{\prime \prime}\left(t_{2}^{\prime}\right) \ldots \phi_{1}^{\prime \prime}\left(t_{n-1}^{\prime}\right) \\
\ldots \ldots \ldots \ldots \ldots \\
\phi_{n-1}^{\prime \prime}\left(t_{2}^{\prime}\right) \ldots \phi_{n-1}^{\prime \prime}\left(t_{n-1}^{\prime}\right)
\end{array}\right| k \\
& =\left|\begin{array}{cccc}
\phi_{1}^{\prime \prime}\left(t_{1}\right) & \phi_{1}^{\prime \prime}\left(t_{2}^{\prime}\right) & \ldots & \phi_{1}^{\prime \prime}\left(t_{n-1}^{\prime}\right) \\
\vdots & \vdots & & \vdots \\
\phi_{n-1}^{\prime \prime}\left(t_{1}\right) & \phi_{n-1}^{\prime \prime}\left(t_{2}^{\prime}\right) & \ldots & \phi_{n-1}^{\prime \prime}\left(t_{n-1}^{\prime}\right)
\end{array}\right|
\end{aligned}
$$

and the non-vanishing can again be ensured by (2.1).

\section{Decoupling inequality for Curves}

Next, we use (1.3) to derive a decoupling inequality for curves (a variant of this approach appears in B-D2].

Let $\Gamma_{1}, \ldots, \Gamma_{n-1} \subset \Gamma \subset \mathbb{R}^{n}$ be as in $\S 2$. Let $\delta>0$ and denote by $\Gamma_{j}^{\delta}$ a $\delta$-neighborhood of $\Gamma_{j}$.

Assume supp $\widehat{f}_{j} \subset \Gamma_{j}^{\delta}$.

Write with $x=\left(x_{0}, x_{1}, \ldots, x_{n-1}\right) \in \mathbb{R}^{n}$ and $\Phi$ as above

$$
\begin{aligned}
& \prod_{j=1}^{n-1}\left[\int_{I_{j}} \widehat{f}_{j}\left(t_{j}\right) e\left(\left\langle\Phi\left(t_{j}\right) \cdot x\right\rangle\right) d t_{j}\right]= \\
& \int_{I_{1}} \cdots \int_{I_{n-1}}\left[\prod_{j=1}^{n-1} \widehat{f}_{j}\left(t_{j}\right)\right] e\left(\left\langle\Phi\left(t_{1}\right)+\cdots+\Phi\left(t_{n-1}\right) \cdot x\right\rangle\right) d t_{1} \ldots d t_{n-1}= \\
& \int_{S}\left[\prod_{j=1}^{n-1} \widehat{f}_{j}\left(t_{j}\right)\right] e(\xi \cdot x) \Omega(\xi) d \xi
\end{aligned}
$$

with $\Omega$ some smooth density on $S$. 
Let $p=\frac{2(n+1)}{n-1}$ and apply the decoupling inequality for $S$ stated in (1.3) of Section 1. Observe that by the regularity of $D_{1}$ in (2.5), a partition of $S$ in $\sqrt{\delta}$-caps $\tau_{\alpha} \subset S$ is equivalent to a partition of $I_{1} \times \cdots \times I_{n-1}$ in $\sqrt{\delta}$-cubes. Hence, denoting by $J \subset[0,1] \sqrt{\delta}$-intervals, we obtain $\|(\underline{3.1})\|_{L^{p}\left(B_{\frac{1}{\delta}}\right)} \ll$

$\delta^{-\frac{n-1}{2(n+1)}-\varepsilon}\left\{\sum_{\substack{J_{1}, \ldots, J_{n-1} \\ J_{j} \subset I_{j}}}\left\|\prod_{j=1}^{n-1}\left[\int_{I_{j}} \widehat{f}_{j}\left(t_{j}\right) e\left(t_{j} x_{0}+f_{1}\left(t_{j}\right) x_{1}+\cdots+f_{n-1}\left(t_{j}\right) x_{n-1}\right) d t_{j}\right]\right\|_{L^{p}\left(B_{\frac{1}{\delta}}\right)}^{p}\right\}^{1 / p}$.

Next take $N=\frac{1}{\delta}$ and discretize inequality (3.2) by setting $t=\frac{k}{N}, k \in$ $\left\{\frac{N}{2}, \ldots, N\right\}$.

This leads to the following inequality for separated intervals $U_{1}, \ldots, U_{n-1} \subset$ $\left\{\frac{N}{2}, \ldots, N\right\}$

$$
\begin{aligned}
& \left\|\prod_{j=1}^{n-1}\left|\sum_{k \in U_{j}} a_{k} e\left(k x_{0}+N \varphi_{1}\left(\frac{k}{N}\right) x_{1}+\cdots+N \varphi_{n-1}\left(\frac{k}{N}\right) x_{n-1}\right)\right|\right\|_{L^{p}\left([0,1]^{n}\right)} \ll \\
& N^{\frac{n-1}{2(n+1)}+\varepsilon}\left(\sum_{\substack{V_{1}, \ldots, V_{n-1} \\
V_{j} \subset U_{j}}}\left\|\prod_{j=1}^{n-1}\left|\sum_{k \in V_{j}} a_{k} e(\cdots)\right|\right\|_{L^{p}\left([0,1]^{n}\right)}^{p}\right)^{\frac{1}{p}}
\end{aligned}
$$

with $V \subset\left\{\frac{N}{2}, \ldots, N\right\}$ running in a partition in $\sqrt{N}$-size intervals.

Note that the domain $[0,1]^{n}$ may always be replaced by a larger box $\prod_{j=0}^{n-1}\left[0, K_{j}\right], K_{j} \geq 1$. In particular, the function

$$
k x_{0}+N \phi_{1}\left(\frac{k}{N}\right) x_{1}+\cdots+N \phi_{n-1}\left(\frac{k}{N}\right) x_{n-1}
$$

in (3.3) may be replaced by

$$
k x_{0}+N_{1} \phi_{1}\left(\frac{k}{N}\right) x_{1}+\cdots+N_{j-1} \phi_{n-1}\left(\frac{k}{N}\right) x_{n-1} \text { where } N_{1}, \ldots, N_{j-1} \geq N \text {. }
$$

Take $\phi_{1}(t)=t^{2}, N_{1}=N^{2}, N_{2}=\cdots=N_{j-1}=N$. We obtain

$$
\begin{aligned}
& \left\|\prod_{j=1}^{n-1}\left|\sum_{k \in U_{j}} e\left(k x_{0}+k^{2} x_{1}+N \phi_{2}\left(\frac{k}{N}\right) x_{2}+\cdots+N \phi_{n-1}\left(\frac{k}{N}\right) x_{n-1}\right)\right|\right\|_{\left.L^{p}([0,1])^{n}\right)} \\
& \ll N^{\frac{n-1}{2(n+1)}+\varepsilon}\left(\sum_{V_{1}, \ldots, V_{n-1}}\left\|\prod_{j=1}^{n-1}\left|\sum_{k \in V_{j}} e(\cdots)\right|\right\|_{L^{p}\left([0,1]^{n}\right)}^{p}\right)^{\frac{1}{p}}
\end{aligned}
$$


Our next task is to bound the individual summands in (3.4).

Write $\bar{k}=\left(k_{1}, \ldots, k_{n-1}\right) \in V_{1} \times \cdots \times V_{n-1}$ as $\bar{k}=\bar{\ell}+\bar{m}, \ell_{j}$ the center of $V_{j}$ and $\left|m_{j}\right|<\sqrt{N}$. Hence

$$
\begin{aligned}
& \sum_{j=1}^{n-1}\left(k_{j} x_{0}+k_{j}^{2} x_{1}+N \phi_{2}\left(\frac{k_{j}}{N}\right) x_{2}+\cdots+N \phi_{n-1}\left(\frac{k_{j}}{N}\right) x_{n-1}\right)= \\
& m_{1}\left(x_{0}+2 \ell_{1} x_{1}+\phi_{2}^{\prime}\left(\frac{\ell_{1}}{N}\right) x_{2}+\cdots+\phi_{n-1}^{\prime}\left(\frac{\ell_{1}}{N}\right) x_{n-1}\right)+ \\
& \vdots \\
& +m_{n-1}\left(x_{0}+2 \ell_{n-1} x_{1}+\phi_{2}^{\prime}\left(\frac{\ell_{n-1}}{N}\right) x_{2}+\cdots+\phi_{n-1}^{\prime}\left(\frac{\ell_{n-1}}{N}\right) x_{n-1}\right)+ \\
& \left(m_{1}^{2}+\cdots+m_{n-1}^{2}\right) x_{1}+\psi(\bar{m}, x)
\end{aligned}
$$

where $|\psi(\bar{m}, x)|<o(1)$ and $\left|\partial_{m} \psi(\bar{m}, x)\right|<O\left(N^{-\frac{1}{2}}\right)$ since $|\bar{m}|<\sqrt{N}$ and $|x|<1$.

Thus $\psi(\bar{m}, x)$ may be dismissed in (3.4) when evaluating $\left\|\prod_{j=1}^{n-1}\left|\sum_{k \in V_{j}} e(\cdots)\right|\right\|_{L^{p}\left([0,1]^{n}\right)}$. Make an affine change of variables

$$
\left(\begin{array}{c}
y_{1} \\
\vdots \\
y_{n-1}
\end{array}\right)=A\left(\begin{array}{c}
x_{0} \\
x_{2} \\
\vdots \\
x_{n-1}
\end{array}\right) \text { with } A=\left[\left(\begin{array}{cccc}
1 & \phi_{2}^{\prime}\left(\frac{\ell_{1}}{N}\right) & \cdots & \phi_{n-1}^{\prime}\left(\frac{\ell_{1}}{N}\right) \\
\vdots & \vdots & & \vdots \\
1 & \phi_{2}^{\prime}\left(\frac{\ell_{n-1}}{N}\right) & \cdots & \phi_{n-1}^{\prime}\left(\frac{\ell_{n-1}}{N}\right)
\end{array}\right)\right]
$$

in the $\left(x_{0}, x_{2}, \ldots, x_{n-1}\right)$ variables, noting that this linear coordinate change can be assumed regular provided $W\left(\phi_{2}^{\prime \prime}, \ldots, \phi_{n-1}^{\prime \prime}\right) \neq 0$ (which is implied by (2.1) for $\left.\phi_{1}(t)=t^{2}\right)$.

Next, using periodicity, another coordinate shift leads to

$$
\begin{aligned}
& \left\|\prod_{j=1}^{n-1}\left|\sum_{k \in V_{j}} e(\cdots)\right|\right\|_{L^{p}\left([0,1]^{n}\right)} \sim \\
& \left\|\sum_{m_{1}, \ldots, m_{n-1}<\sqrt{N}} e\left(m_{1} y_{1}+\cdots+m_{n-1} y_{n-1}+\left(m_{1}^{2}+\cdots+m_{n-1}^{2}\right) x_{1}\right)\right\|_{L_{x_{1}, y_{1}, \ldots, y_{n-1}}^{p}\left([0,1]^{n}\right)} \\
& \lll N^{\frac{n-1}{4}+\varepsilon}
\end{aligned}
$$


by the Strichartz inequality on $\mathbb{T}^{n}$.

Summarizing, we proved the following multi-linear mean value theorem.

Theorem 1. Assume $n \geq 3$ and $\varphi_{2}, \ldots, \varphi_{n-1}$ satisfying

$$
W\left(\varphi_{2}^{\prime \prime \prime}, \ldots, \varphi_{n-1}^{\prime \prime \prime}\right) \neq 0 \text {. }
$$

Let $U_{1}, \ldots, U_{n-1} \subset\left[\frac{N}{2}, N\right] \cap \mathbb{Z}$ be $O(N)$-separated intervals. Then

$$
\begin{aligned}
& \left\|\prod_{j=1}^{n-1}\left|\sum_{k \in U_{j}} e\left(k x_{0}+k^{2} x_{1}+N \varphi_{2}\left(\frac{k}{N}\right) x_{2}+\cdots+N \varphi_{n-1}\left(\frac{k}{N}\right) x_{n-1}\right)\right|\right\|_{L^{\frac{2(n+1)}{n-1}\left([0,1]^{n}\right)}} \leq \\
& N^{\frac{n-1}{2}+\varepsilon} .
\end{aligned}
$$

\section{Remarks.}

(i) Theorem 1 remains valid (following the same argument) with coefficients $a_{k}, k \in U_{j}$ and r.h.s. replaced by $\prod_{j=1}^{n-1}\left(\sum_{k \in U_{j}}\left|a_{k}\right|^{2}\right)^{\frac{1}{2}}$.

(ii) Note that (3.7) is best possible. Indeed, restricting $\left|x_{0}\right|<\frac{1}{N},\left|x_{1}\right|<$ $\frac{1}{N^{2}},\left|x_{2}\right|<\frac{1}{N}, \ldots,\left|x_{n-1}\right|<\frac{1}{N}$, one gets the contribution

$$
N^{n-1-\frac{n-1}{2(n+1)}(n+1)}=N^{\frac{n-1}{2}} .
$$

(iii) Also, as we will see shortly, (3.7) is only valid in the above multi-linear form.

\section{Mean values estimates for the 8th and 10th moment}

Note that (3.7) is the multi-linear version of an estimate on

$$
\left\|\sum_{k \sim N} e\left(k x_{0}+k^{2} x_{1}+N \varphi_{2}\left(\frac{k}{N}\right) x_{2}+\cdots+N \varphi_{n-1}\left(\frac{k}{N}\right) x_{n-1}\right)\right\|_{L^{2(n+1)}\left([0,1]^{n}\right)} \text {. }
$$

Denoting $f_{I}=\sum_{k \in I} e\left(k x_{0}+k^{2} x_{1}+N \varphi_{2}\left(\frac{k}{N}\right) x_{2}+\cdots+N \varphi_{n-1}\left(\frac{k}{N}\right) x_{n-1}\right)$ for $I \subset[1, N]$ a subinterval, one adopts the following argument from [B-G]. Partition $[1, N]$ in intervals $I$ of size $N^{1-\tau}(\tau>0$ small). Fix a point $x$ and distinguish the following two scenarios. Either we can find $n-1$ intervals $I_{1}, \ldots, I_{n-1}$ that are $O\left(N^{1-\tau}\right)$-separated and such that

$$
\left|f_{I_{j}}(x)\right|>N^{-2 \tau}|f(x)| \text { for } 1 \leq j \leq n-1
$$

or for some interval $I$, we have

$$
\left|f_{I}(x)\right|>c|f(x)|
$$


The contribution of (4.2) is captured by the multi-linear estimate (3.7) and we obtain $N^{\frac{1}{2}+c \tau}$. For the (4.3)-contribution, bound by

$$
\max \left|f_{I}\right| \leq\left[\sum_{I}\left|f_{I}\right|^{2(n+1)}\right]^{\frac{1}{2(n+1)}}
$$

contributing to

$$
\left[\sum_{I}\left\|f_{I}\right\|_{2(n+1)}^{2(n+1)}\right]^{\frac{1}{2(n+1)}} .
$$

One may then repeat the process to each $f_{I}$. Note that after a coordinate change in $x_{0}, x_{1}$, we obtain exponential sums of the form

$F(x)=\sum_{\ell \sim M} e\left(\ell x_{0}+\ell^{2} x_{1}+N \varphi_{2}\left(\frac{k}{N}+\frac{\ell}{N}\right) x_{2}+\cdots+N \varphi_{n-1}\left(\frac{k}{N}+\frac{\ell}{N}\right) x_{n-1}\right)$

with $M=N^{1-\tau}, k \sim N$ fixed. Set for $j=1, \ldots, n-1$.

$$
N \varphi_{j}\left(\frac{k}{N}+\frac{\ell}{N}\right)=M \psi_{j}\left(\frac{\ell}{M}\right)
$$

with

$$
\psi_{j}(t)=\frac{N}{M} \varphi\left(\frac{k}{N}+\frac{M}{N} t\right) .
$$

However the Wronskian condition $W\left(\psi_{2}^{\prime \prime \prime}, \ldots, \psi_{n-1}^{\prime \prime \prime}\right)>O(1)$ deteriorated. For $n=3$ we will nevertheless be able to retrieve easily the expected bound, while for $n \geq 4$, the linear bounds turn out to be weaker than the multilinear one.

Let $n=3$. Then $\psi_{2}^{\prime \prime \prime}(t)=\frac{M^{2}}{N^{2}} \varphi^{\prime \prime \prime}\left(\frac{k}{N}+\frac{M}{N} t\right)=O\left(\frac{M^{2}}{N^{2}}\right)$ and replacing $\psi_{2}=\frac{M^{2}}{N^{2}} \tilde{\varphi}_{2}, x_{2}^{\prime}=\frac{M^{2}}{N^{2}} x_{2}$, this leads to

$$
\begin{aligned}
\|F\|_{L_{x_{0}, x_{1}, x_{2}=O(1)}^{8}} & \sim\left(\frac{N}{M}\right)^{\frac{1}{4}}\left\|\sum_{\ell \sim M} e\left(\ell x_{0}+\ell^{2} x_{1}+M \tilde{\varphi}_{2}\left(\frac{\ell}{M}\right) x_{2}^{\prime}\right)\right\|_{\substack{L_{x_{0}, x_{1}=O(1)}^{8} \\
x_{2}^{\prime}=O\left(\frac{M^{2}}{N^{2}}\right)}} \\
& \leq\left(\frac{N}{M}\right)^{\frac{1}{4}}\|\cdots\|_{L_{x_{0}, x_{1}, x_{2}}^{8}=O(1)} \\
& <\left(\frac{N}{M}\right)^{\frac{1}{4}} M^{\frac{1}{2}+\varepsilon}
\end{aligned}
$$

assuming the expected bound at scale $M$. The bound on (4.4) becomes then

$$
\left(\frac{N}{M}\right)^{\frac{3}{8}} M^{\frac{1}{2}+\varepsilon}=\left(\frac{M}{N}\right)^{\frac{1}{8}} N^{\frac{1}{2}+\varepsilon}
$$

and summing over dyadic $M<N$ we reproved the main result from [B-I2]. 
Theorem 2. B-I2.

Assume $\varphi^{\prime \prime \prime} \neq 0$. Then

$$
\left\|\sum_{k \sim N} e\left(k x_{0}+k^{2} x_{1}+N \varphi\left(\frac{k}{N}\right) x_{2}\right)\right\|_{8} \ll N^{\frac{1}{2}+\varepsilon} .
$$

Note that in their application to $\zeta\left(\frac{1}{2}+i t\right), \varphi(t)=t^{3 / 2}$.

It is interesting to note that unlike in B-I2, our derivation of (4.8) did not make use of Poisson summation (i.e. Process B).

The work of [B-I1] was extensively refined by Huxley and his collaborators, resulting in his book $[\mathrm{H}]$.

The present discussion is relevant to the so called 'First Spacing Problem'; (4.8) indeed means that the system

$$
\text { (4.9) }\left\{\begin{array}{l}
k_{1}+k_{2}+k_{3}+k_{4}=k_{5}+\cdots+k_{8} \\
k_{1}^{2}+\cdots+k_{4}^{2}=k^{2}+\cdots+k_{8}^{2} \\
k_{1}^{3 / 2}+\cdots+k_{4}^{3 / 2}=k_{5}^{3 / 2}+\cdots+k_{8}^{3 / 2}+O(\sqrt{N}) .
\end{array}\right.
$$

has at most $N^{4+\varepsilon}$ solutions in integers $k_{1}, \ldots, k_{8} \sim N$ (the statement is clearly optimal).

Huxley considers the more elaborate problem in 10-variables

$$
\text { (4.10) }\left\{\begin{array}{l}
k_{1}+k_{5}=k_{6}+\cdots+k_{10} \\
k_{1}^{2}+\ldots+k_{5}^{2}=k_{6}^{2}+\cdots+k_{10}^{2} \\
k_{1}^{3 / 2}+\cdots+k_{5}^{3 / 2}=k_{6}^{3 / 2}+\cdots+k_{10}^{3 / 2}+O\left(\delta N^{3 / 2}\right) \\
k_{1}^{1 / 2}+\cdots+k_{5}^{1 / 2}=k_{6}^{1 / 2}+\cdots+k_{10}^{1 / 2}+O\left(\Delta N^{\frac{1}{2}}\right)
\end{array}\right.
$$

(see $[\mathrm{H}], \S 11)$ for which the number $N_{10}(\delta, \Delta)$ of solutions is given by the 10th moment

$$
\left\|\sum_{k \sim N} e\left(k x_{0}+k^{2} x_{1}+\frac{1}{\delta}\left(\frac{k}{N}\right)^{3 / 2} x_{2}+\frac{1}{\Delta}\left(\frac{k}{N}\right)^{1 / 2} x_{3}\right)\right\|_{L_{x_{0}, x_{1}, x_{2}, x_{3}}^{10}}^{10} .
$$

In the applications to exponential sums, $\Delta=\delta N, \frac{1}{N^{2}}<\delta<\frac{1}{N}$. In this setting, the following key inequality appears in [H-K].

Theorem 3. [H-K]. With $\Delta=\delta N, \frac{1}{N^{2}}<\delta<\frac{1}{N}$, we have

$$
N_{10}(\delta, \delta N) \ll \delta . N^{7+\varepsilon} .
$$


In what follows, we estimate (4.11) using Theorem 7 and will in particular retrieve (4.12) in a stronger form.

Start by observing that, as a consequence of (3.7), for $U_{1}, U_{2}, U_{3}$ and $\varphi_{2}, \varphi_{3}$ as in Theorem 1

$$
\begin{gathered}
\int_{0}^{1} \int_{0}^{1} \int_{0}^{1} \int_{0}^{1}\left\{\prod_{j=1}^{3} \mid \sum_{k \in U_{j}} e\left(k x_{0}+k^{2} x_{1}+\frac{1}{\delta} \varphi_{2}\left(\frac{k}{N}\right) x_{2}+\left.\frac{1}{\Delta} \varphi_{3}\left(\frac{k}{N}\right) x_{3}\right|^{\frac{10}{3}}\right\} d x_{0} d x_{1} d x_{2} d x_{3} \ll\right. \\
{[\min (\delta N, N)+1][\min (\Delta N, N)+1] N^{5+\varepsilon}}
\end{gathered}
$$

Using the scale reduction described in (4.1)-(4.7), we also need to evaluate the contributions of

$$
\frac{N}{M} \cdot(4.14)
$$

with

$$
(4.14)=\int_{0}^{1} \int_{0}^{1} \int_{0}^{1} \int_{0}^{1}\left\{\prod_{j=1}^{3} \mid \sum_{\ell \in U_{j}} e\left(\ell x_{0}+\ell^{2} x_{1}+\frac{1}{\delta} \varphi_{2}\left(\frac{k+\ell}{N}\right) x_{2}+\left.\frac{1}{\Delta} \varphi_{3}\left(\frac{k+\ell}{N}\right) x_{3}\right|^{\frac{10}{3}}\right\}\right.
$$

where $k \in\left[\frac{N}{2}, N\right], I=\left[k, k+M\left[\right.\right.$ and $U_{1}, U_{2}, U_{3}$ are $\sim M$ separated subintervals of size $\sim M$ in $I$. By a change of variables in $x$, the phase function in (4.16) may be replaced by

$$
e\left(\ell x_{0}+\ell^{2} x_{1}+\frac{M^{3}}{\delta N^{3}} \tilde{\varphi}_{2}\left(\frac{\ell}{M}\right) x_{2}+\frac{M^{4}}{\Delta N^{4}} \tilde{\varphi}_{3}\left(\frac{\ell}{M}\right) x_{3}\right)
$$

where $\tilde{\varphi}_{2}(t)$ has leading monomial $t^{3}$ and $\tilde{\varphi}_{3}(t)$ leading monomial $t^{4}$. Hence $W\left(\tilde{\varphi}_{2}^{\prime \prime \prime}, \tilde{\varphi}_{3}^{\prime \prime \prime}\right)>c$ and (4.13) is applicable to (4.14) with $N, \delta, \Delta$ replaced by $M, \frac{\delta N^{3}}{M^{3}}, \frac{\Delta N^{4}}{M^{4}}$. Therefore

$$
\left(\underline{4.15)} \ll\left[1+\min \left(\frac{\delta N^{3}}{M^{2}}, M\right)\right]\left[1+\min \left(\frac{\Delta N^{4}}{M^{3}}, M\right)\right] M^{4} N^{1+\varepsilon}\right.
$$

and (4.17) needs to be summed over dyadic $M<N$. One easily checks that the conclusion is as follows

Theorem 4. Assume $W\left(\varphi_{2}^{\prime \prime \prime}, \varphi_{3}^{\prime \prime \prime}\right) \neq 0$ and $\delta<\Delta$. Then

$$
\begin{aligned}
& \left\|\sum_{k \sim N} e\left(k x_{0}+k^{2} x_{1}+\frac{1}{\delta} \varphi_{2}\left(\frac{k}{N}\right) x_{2}+\frac{1}{\Delta} \varphi_{3}\left(\frac{k}{N}\right) x_{3}\right)\right\|_{10}^{10} \ll \\
& {\left[\delta \Delta^{3 / 4} N^{7}+(\delta+\Delta) N^{6}+N^{5}\right] N^{\varepsilon} .}
\end{aligned}
$$

In particular

$$
N_{10}(\delta, \Delta)<(4.18)
$$


Hence, we are retrieving Theorem 3.

Remark. We make the following comment on the role of the first term in the r.h.s. of (4.18), relevant to the Remark following Theorem 1.

Partition $\left[\frac{N}{2}, N\right]$ in intervals $I=[n, n+M]$ of size $M$. Obviously $N_{10}(\delta, \Delta)$ is at least $\frac{N}{M}$ times a lower bound on the number of solutions of

$$
\left\{\begin{array}{l}
m_{1}+\cdots+m_{5}=m_{6}+\cdots+m_{10} \\
m_{1}^{2}+\cdots+m_{5}^{2}=m_{6}^{2}+\cdots+m_{10}^{2} \\
\left(\frac{n+m_{1}}{N}\right)^{\frac{3}{2}}+\cdots+\left(\frac{n+m_{5}}{N}\right)^{\frac{3}{2}}=\left(\frac{n+m_{6}}{N}\right)^{\frac{3}{2}}+\cdots+\left(\frac{n+m_{10}}{N}\right)^{\frac{3}{2}}+O(\delta) \\
\left(\frac{n+m_{1}}{N}\right)^{\frac{1}{2}}+\cdots+\left(\frac{n+m_{5}}{N}\right)^{\frac{1}{2}}=\left(\frac{n+m_{6}}{N}\right)^{\frac{1}{2}}+\cdots+\left(\frac{n+m_{10}}{N}\right)^{\frac{1}{2}}+O(\Delta)
\end{array}\right\}
$$

Since

$$
\begin{aligned}
& \left(\frac{n+m}{N}\right)^{\frac{3}{2}}=\left(\frac{n}{N}\right)^{\frac{3}{2}}+\frac{3}{2}\left(\frac{n}{N}\right)^{\frac{1}{2}} \frac{m}{N}+\frac{3}{2}\left(\frac{n}{N}\right)^{-\frac{1}{2}}\left(\frac{m}{N}\right)^{2}-\frac{1}{16}\left(\frac{n}{N}\right)^{-\frac{3}{2}}\left(\frac{m}{N}\right)^{3}+\ldots \\
& \left(\frac{n+m}{N}\right)^{\frac{1}{2}}=\left(\frac{n}{N}\right)^{\frac{1}{2}}+\frac{1}{2}\left(\frac{n}{N}\right)^{-\frac{1}{2}} \frac{m}{N}-\frac{1}{8}\left(\frac{n}{N}\right)^{-\frac{3}{2}}\left(\frac{m}{N}\right)^{2}+\frac{3}{16}\left(\frac{n}{N}\right)^{-\frac{5}{2}}\left(\frac{m}{N}\right)^{3}-\frac{15}{128}\left(\frac{n}{N}\right)^{-\frac{7}{2}}\left(\frac{m}{N}\right)^{4}+\ldots
\end{aligned}
$$

the equations (4.20) may be replaced by

$$
\left\{\begin{array}{l}
\varphi\left(\frac{m_{1}}{N}\right)+\cdots-\varphi\left(\frac{m_{10}}{N}\right)=O(\delta) \\
\psi\left(\frac{m_{1}}{N}\right)+\cdots-\psi\left(\frac{m_{10}}{N}\right)=O(\Delta)
\end{array}\right.
$$

with $\varphi, \psi$ of the form $\varphi(t)=a_{3} t^{3}+a_{4} t^{4}+\cdots$ and $\psi(t)=b_{3} t^{3}+b_{4} t^{4}+\cdots$ and where $\left|\begin{array}{ll}a_{3} & b_{3} \\ a_{4} & b_{4}\end{array}\right| \neq 0$.

Assume $\delta<\Delta$ and replace $\psi$ by $\psi_{1}=\psi-\frac{b_{3}}{a_{3}} \varphi=c_{4} t^{4}+\cdots$ Writing $\frac{m}{N}=\frac{M}{N} \frac{m}{M}$, we obtain conditions of the form

$$
\left\{\begin{array}{l}
\tilde{\varphi}\left(\frac{m_{1}}{M}\right)+\cdots-\tilde{\varphi}\left(\frac{m_{10}}{N}\right)<O\left(\frac{N^{3}}{M^{3}} \delta\right) \\
\tilde{\psi}_{1}\left(\frac{m_{1}}{M}\right)+\cdots-\tilde{\psi}_{1}\left(\frac{m_{10}}{N}\right)<O\left(\frac{N^{4}}{M^{4}} \Delta\right)
\end{array}\right.
$$

where $\tilde{\varphi}=t^{3}+\cdots, \tilde{\psi}_{1}=t^{4}+\cdots$. Consider the system $(4.19)+(4.21)$ with $m_{i} \leq M$. Clearly the number of solutions is at least

$$
M^{7} \min \left(1, \frac{N^{3}}{M^{3}} \delta\right) \cdot \min \left(1, \frac{N^{4}}{M^{4}} \Delta\right) .
$$

Taking $M=\Delta^{1 / 4} N$, we obtain $N^{7} \delta \Delta$. The quantity is multiplied further with $\frac{N}{M}$, leading to a lower bound $\delta \Delta^{3 / 4} N^{7}$ for $N_{10}(\delta, \Delta)$. 
This shows that the first term in (4.18) (apart from the $N^{\varepsilon}$ factor) is also a lower bound.

In our applications, $\Delta$ tends to be much larger then $\delta$ which makes $\Delta N$ the leading term in (4.18). Next, we develop an argument to reduce the weight of $\Delta N$ by involving also some ideas and techniques from $[\mathrm{H}]$. It is likely that our presentation can be improved at this point.

We will need the following variant of van der Corput's exponential sum bound (cf. [Ko], Theorem 2.6).

Lemma 5. Assume $f$ a smooth function on $I=\left[\frac{N}{2}, N\right]$ and $f^{(3)} \sim \lambda_{3}$. Let $\left\{V_{j}\right\}$ denote a partition of $I$ in intervals of size $D$. Then

$$
\sum_{j}\left|\sum_{n \in V_{j}} e(f(n))\right|^{2} \lesssim\left\{\begin{array}{l}
N+D^{\frac{1}{2}} \lambda_{3}^{-\frac{1}{2}}+D^{\frac{3}{2}} \lambda_{3}^{\frac{1}{2}} N \\
N D \lambda_{3}^{\frac{1}{3}}+D \lambda_{3}^{-\frac{1}{3}} \text { if } D>\lambda_{3}^{-\frac{1}{3}} .
\end{array}\right.
$$

We first proceed with a multi-linear bound considering instead of (4.13) 5-linear expressions with $U_{j} \subset\left[\frac{N}{2}, N\right]$ of size $\sim N$ and $\sim N$ separated $(1 \leq j \leq 5)$

$$
\int\left\{\prod_{j=1}^{5}\left|\sum_{k \in U_{j}} e\left(k x_{0}+k^{2} x_{1}+\frac{1}{\delta}\left(\frac{k}{N}\right)^{3 / 2} x_{2}+\frac{1}{\Delta}\left(\frac{k}{N}\right)^{\frac{1}{2}} x_{3}\right)\right|^{2}\right\} d x_{0} d x_{1} d x_{2} d x_{3}
$$

This quantity will increase by increasing $\delta$ and we replace $\delta$ by a parameter $\delta_{1}>\delta$ to be specified. An application of Hölder's inequality permits then to bound (4.24) by (4.13) with $\delta$ replaced by $\delta_{1}$.

Assuming $\Delta<1$, perform a decoupling at scale $N \Delta^{\frac{1}{2}}$ using (3.2). This gives an estimate on the 1.h.s. of (4.13) by

$$
\Delta^{-1} \int \prod_{j=1}^{3}\left[\sum_{V_{j} \subset U_{j}}\left|\sum_{k \in V_{j}} e(\cdots)\right|^{\frac{10}{3}}\right] d x
$$

with $V_{j} \subset U_{j}$ a partition in $N \Delta^{\frac{1}{2}}$-intervals. Using again Hölder's inequality, one may bound

$$
\prod_{j=1}^{3}\left[\sum_{V_{j} \subset U_{j}}||^{\frac{10}{3}}\right] \leq\left(\sum_{V \subset\left[\frac{N}{2}, N\right]}||^{2}\right) \prod_{j=1}^{2}\left(\sum_{V_{j} \subset U_{j}}||^{4}\right)+\cdots
$$

where $\cdots$ refers to the pairs $U_{2}, U_{3}$ and $U_{3}, U_{1}$ instead of $U_{1}, U_{2}$. 
Specifying in (4.13), with $\delta$ replaced by $\delta_{1}$, a range

$$
x_{2} \sim X_{2}<1 \text { assuming } X_{2} \Delta>100 \delta_{1}
$$

an application of (4.22) to the first factor of (4.26) with $D=\Delta^{\frac{1}{2}} N, \lambda_{3} \sim$ $\frac{X_{2}}{\delta_{1} N^{3}}$ gives the bound

$$
N+\delta_{1}^{\frac{1}{2}} \Delta^{\frac{1}{4}} N^{2} X_{2}^{-\frac{1}{2}}+\delta_{1}^{-\frac{1}{2}} \Delta^{\frac{3}{4}} N X_{2}^{\frac{1}{2}} .
$$

We always assume

$$
\Delta N>100
$$

(this condition remains clearly preserved at lower scales, cf. (4.17)).

Apply the bilinear estimate (Theorem 1 with $n=3$ ) to the second factor of (4.26) considering the variables $x_{0}, x_{1}, x_{2}$ and restricting $x_{2} \sim X_{2}$. By (4.24), this gives the contribution

$$
\left(1+\delta_{1}^{\frac{1}{2}} \Delta^{\frac{1}{4}} N X_{2}^{-\frac{1}{2}}+\delta_{1}^{-\frac{1}{2}} \Delta^{\frac{3}{4}} X_{1}^{\frac{1}{2}}\right)\left(X_{2}+\delta_{1} N\right) N^{5+\varepsilon} .
$$

Assuming $X_{2}>\delta_{1} N$, which by (4.29) implies (4.27), gives the bound

$$
\left(1+\delta_{1}^{\frac{1}{2}} \Delta^{\frac{1}{4}} N+\delta_{1}^{-\frac{1}{2}} \Delta^{\frac{3}{4}}\right) N^{5+\varepsilon} .
$$

The contribution of $X_{2}<\delta_{1} N$ is estimated by

$$
\begin{gathered}
\Delta^{-1} \int_{0}^{1} \int_{0}^{1} \int_{0}^{\delta_{1} N} \int_{0}^{1}\left\{\prod_{j=1}^{3}\left[\sum_{V_{j} \subset U_{j}}||^{\frac{10}{3}}\right]\right\} d x_{0} d x_{1} d x_{2} d x_{3} \\
\ll \Delta^{-1}\left(\frac{N}{\Delta^{1 / 2} N}\right)^{3}(\Delta N) \delta_{1} N\left(\Delta^{\frac{1}{2}} N\right)^{5} \ll \Delta \delta_{1} N^{7+\varepsilon}
\end{gathered}
$$

assuming $\delta_{1} N<1$. This gives

$$
\left(1+\delta_{1}^{-\frac{1}{2}} \Delta^{\frac{3}{4}}+\delta_{1}^{\frac{1}{2}} \Delta^{\frac{1}{4}} N+\Delta \delta_{1} N^{2}\right) N^{5+\varepsilon}
$$

and setting $\delta_{1}=\Delta^{\frac{1}{2}} N^{-1}$, assuming $\delta_{1}>\delta$ gives

$$
\left((\Delta N)^{\frac{1}{2}}+\Delta^{3 / 2} N\right) N^{5+\varepsilon} .
$$

If $\delta_{1} \leq \delta$, use (4.30) with $\delta_{1}=\delta$.

Thus the multi-linear contribution in the 10th moment may be estimated by

$$
\left(\delta^{\frac{1}{2}} \Delta^{\frac{1}{4}} N+\Delta \delta N^{2}+(\Delta N)^{\frac{1}{2}}+\Delta^{3 / 2} N\right) N^{5+\varepsilon}
$$


Next, consider te lower scale contributions

$$
\sum_{\substack{I \subset\left[\frac{N}{2}, N\right] \\|I|=M}}\left\|\sum_{n \in I} e(\cdots)\right\|_{10}^{10} .
$$

Fixing $M<N$ and replacing $\delta$, resp. $\Delta$, by $\frac{N^{3}}{M^{3}} \delta$, resp. $\frac{N^{4}}{M^{4}} \Delta$, we obtain the bound

$$
\left(\delta^{\frac{1}{2}} \Delta^{\frac{1}{4}} N+(\Delta N)^{\frac{1}{2}}\right) N^{5+\varepsilon}+\left(\Delta \delta N+\Delta^{3 / 2}\right) \frac{N^{7+\varepsilon}}{M}
$$

for the multi-linear contribution at scale $M$.

On the other hand, we can also make a crude estimate using the $L^{8}$-norm, leading to the contribution

$$
\frac{N}{M} M^{2}\left(1+\delta \frac{N^{3}}{M^{2}}\right) M^{4+\varepsilon} \ll N M^{5+\varepsilon}+\delta N^{4} M^{3+\varepsilon}
$$

and $(4.34) \ll(\Delta N)^{\frac{1}{2}} N^{5+\varepsilon}$ for $M<(\Delta N)^{\frac{1}{10}} N^{4 / 5}$ and $\delta<N^{-7 / 5}(\Delta N)^{\frac{1}{5}}$.

Hence we get

Lemma 6. For $N \Delta>1$ and $\delta<N^{-7 / 5}(\Delta N)^{\frac{1}{5}}$

$$
N_{10}(\delta, \Delta) \ll\left(\delta^{\frac{1}{2}} \Delta^{\frac{1}{4}} N+(\Delta N)^{\frac{1}{2}}\right) N^{5+\varepsilon}+\left(\Delta \delta N+\Delta^{\frac{3}{2}}\right)(\Delta N)^{-\frac{1}{10}} N^{\frac{31}{5}+\varepsilon} .
$$

Next, recall Lemma 11, 3.3 in $[\underline{\mathrm{H}}]$.

Lemma 7. Assume $\frac{1}{N}>\delta>\frac{1}{N^{2}}$ and $\frac{1}{N}<\Delta<\delta N$. Letting

$$
2 \leq T \leq \frac{1}{\sqrt{\delta N}}
$$

be a parameter, the following inequality holds

$$
N_{10}(\delta, \Delta) \lesssim \frac{1}{T} N_{10}\left(T^{2} \delta, T \Delta\right)+N_{10}(\delta, C T \delta) .
$$

Combining Theorem 4, Lemmas 6 and 7 (applied with $T=\Delta N$ ) gives

Lemma 8. Assume $\frac{1}{N}>\delta>\frac{1}{N^{2}}, \frac{1}{N}<\Delta<\delta N$ and $\Delta \sqrt{\delta} N^{3 / 2}<1$. Then $N_{10}(\delta, \Delta)<\left(1+\delta(\Delta N)^{\frac{8}{5}} N^{\frac{7}{5}}\right)\left(1+\delta^{\frac{1}{2}}(\Delta N)^{\frac{1}{2}} N^{\frac{3}{4}}\right) N^{5+\varepsilon}+\left(\delta(\Delta N)^{\frac{14}{5}}+(\Delta N)^{\frac{3}{10}} \Delta^{\frac{3}{2}}\right) N^{\frac{31}{5}+\varepsilon}$.

Setting $\Delta=\delta N$ leads to the following strengthening of Theorem 3

Theorem 9. For $N^{-\frac{33}{18}} \geq \delta \geq N^{-2}$, we have $N_{10}(\delta, \delta N) \ll N^{5+\varepsilon}$. 
Note that in view of the Remark following Theorem 4, the conclusion of Theorem 9 fails for $\delta>N^{-11 / 7}$.

\section{On an inequality of Robert and Sargos}

In [R-S] established the inequity

$$
I_{10}=\int_{0}^{1} \int_{0}^{1}\left(\left.\sum_{n \sim N} e\left(n^{2} x+n^{4} y\right)\right|^{10} d x d y \ll N^{\frac{49}{8}+\varepsilon}\right.
$$

which they applied to obtain new estimates on Weyl sums. An improvement of (5.1) appears in $[\mathrm{P}$, who obtains

$$
I_{10} \ll N^{6+\varepsilon} .
$$

Using our methods, we present a further improvement.

\section{Theorem 10.}

$$
I_{10} \ll N^{\frac{17}{3}+\varepsilon}
$$

The corresponding improvement in Weyl's inequality following HeathBrown's method was recorded in the Introduction.

Note that bounding $I_{10}$ is tantamount to estimating the number of integral solutions $n_{i} \sim N(1 \leq 1 \leq 10)$ of the system

$$
\left\{\begin{array}{l}
n_{1}^{2}+n_{2}^{2}+n_{3}^{2}+n_{4}^{2}+n_{5}^{2}=n_{6}^{2}+n_{7}^{2}+n_{8}^{2}+n_{9}^{2}+n_{10}^{2} \\
n_{1}^{4}+n_{2}^{4}+n_{3}^{4}+n_{4}^{4}+n_{5}^{4}=n_{6}^{4}+n_{7}^{4}+n_{8}^{4}+n_{9}^{4}+n_{10}^{4} .
\end{array}\right.
$$

The problem is not shift invariant and therefore as it stands not captured by a Vinogradov mean value theorem of the usual kind. Following Wooley's approach for $\left(n, n^{3}\right)$ (see [W]), knowledge of the (conjectural) optimal VMVT for $k=4$ (which would involve the 20th moment) and interpolation with the 6th moment would at the best deliver $I_{10} \ll N^{\frac{41}{7}}$, inferior to (5.3).

A crude summary of our argument. As in [R-S], we need to consider the more general expressions

$$
I_{p}(\lambda)=\int_{0}^{1} \int_{0}^{1}\left|\sum_{n \sim N} e\left(n^{2} x+\lambda n^{4} y\right)\right|^{p} d x d y
$$

with $p \geq 6$ and $0<\lambda \leq 1$. A first step is an application of the decoupling theorem from [B-D] for planar curves similarly as in B-D, Theorem 2.18 (where an extension of the result $I_{6}\left(N^{-3}\right) \ll N^{3+\varepsilon}$ from [R-S] is established). At this stage, one gets shorter sums, of length $M$ say, i.e. $n \in\left[n_{0}, n_{0}+M\right]$ 
with $n_{0}$ ranging in $\left[N, \frac{N}{2}\right]$. Exploiting $n_{0}$ as an additional variable leads then to mean value expressions of the form

$$
\int_{0}^{1} \int_{0}^{1} \int_{0}^{1} \int_{0}^{1}\left|\sum_{m \sim M} e\left(x m+y m^{2}+\lambda N z m^{3}+\lambda w m^{4}\right)\right|^{p} d x d y d z d w
$$

to which Theorem 1 is applicable. In the above $\lambda$ plays the role of a parameter, nothing that $I_{p}(\lambda)$ decreases with $\lambda$ for $p$ an even integer.

\subsection{Preliminary decoupling.}

Denote $S=\sum_{n \sim N} e\left(n^{2} x+\lambda n^{4} y\right)$ and $S_{I}=\sum_{n \in I} e\left(n^{2} x+\lambda n^{4} y\right)$ for $I \subset\left[\frac{N}{2}, N\right]$ an interval. Assuming

$$
\lambda N^{4}>\frac{N^{2}}{M^{2}}, \text { i.e. } \lambda N^{2} M^{2}>1
$$

the decoupling theorem for curves gives for $p \geq 6$

$$
\|S\|_{p} \ll N^{\varepsilon}\left(\frac{N}{M}\right)^{\frac{1}{2}-\frac{3}{p}}\left(\sum_{I}\left\|S_{I}\right\|_{p}^{2}\right)^{\frac{1}{2}}
$$

with $\{I\}$ a partition of $\left[\frac{N}{2}, N\right]$ in $M$-intervals. Hence

$$
I_{p}(\lambda) \ll N^{\varepsilon}\left(\frac{N}{M}\right)^{p-3}\left(\frac{1}{N} \sum_{n \sim N} \int_{0}^{1} \int_{0}^{1}\left|S_{[n, n+M]}(x, y)\right|^{p} d x d y\right)
$$

where

$$
\left|S_{[n, n+M]}(x, y)\right|=\left|\sum_{m \sim M} e\left(\left(2 n x+4 \lambda n^{3} y\right) m+\left(x+6 \lambda n^{2} y\right) m^{2}+4 \lambda n m^{3} y+\lambda m^{4} y\right)\right| .
$$

\subsection{Distributional considerations.}

In view of (5.9), (5.10) and exploiting the additional average over $n$, it is natural to analyze the distribution induced by the map

$$
\begin{array}{r}
\varphi:[0,1] \times[0,1] \times\{n \sim N\} \rightarrow \mathbb{T} \times \mathbb{T} \times[0,4 N] \times[0,1] \\
(x, y, n) \mapsto\left(2 n x+4 \lambda n^{3} y, x+6 \lambda n^{2} y, 4 n y, y\right)=\left(x^{\prime}, y^{\prime}, z^{\prime}, \omega^{\prime}\right) .
\end{array}
$$

For the time, restrict $y$ to $\left[\frac{1}{2}, 1\right]$ and denote $\mu$ the (normalized) image measure of $\varphi$. A translation $x \mapsto x-2 \lambda n^{2} y(\bmod 1)$ clearly permits to replace $\varphi$ by the map

$$
(x, y, n) \mapsto\left(2 n x, x+4 \lambda n^{2} y, 4 n y, y\right)
$$


and we need to analyze the distribution of $\mu$ at scale $\frac{1}{M} \times \frac{1}{M^{2}} \times \frac{1}{\lambda M^{3}} \times \frac{1}{\lambda M^{4}}$.

Hence, let $k, \ell \in \mathbb{Z},|k| \lesssim M,|\ell| \lesssim M^{2}$ and $\xi, \eta \in \mathbb{R},|\xi| \lesssim \lambda M^{3},|\eta|<\lambda M^{4}$. The Fourier transform $\hat{\mu}$ of $\mu$ is given by

$$
\hat{\mu}(k, \ell, \xi, \eta)=\frac{1}{N} \sum_{n \sim N} \iint d x d y e\left(2 n x k+\left(x+4 \lambda n^{2} y\right) \ell+4 n y \xi+y \eta\right)
$$

implying

$$
|\hat{\mu}(k, \ell, \xi, \eta)| \ll \frac{1}{N} \sum_{n \sim N} 1_{[2 n k+\ell=0]} 1_{\left[\left|4 \lambda n^{2} \ell+4 n \xi+\eta\right| \ll N^{\varepsilon}\right]} .
$$

It follows from the restrictions on $\xi, \eta$ that

$$
|\ell| \ll \frac{N^{\varepsilon}}{\lambda N^{2}}+\frac{M^{3}}{N}=\frac{M^{4}}{N^{2}}<\frac{1}{\lambda N^{2-\varepsilon}}+\frac{M^{3}}{N}
$$

and

$$
|k| \ll \frac{1}{\lambda N^{3-\varepsilon}}+\frac{M^{3}}{N^{2}}
$$

Assume further

$$
\lambda>N^{-3+\varepsilon} \text { and } M<N^{\frac{2}{3}-\varepsilon}
$$

as to ensure $k=\ell=0$.

Hence $\mu \ll N^{\varepsilon} \pi_{z^{\prime}, w^{\prime}}[\mu]$. Returning to (5.9), we may therefore bound

$$
\frac{1}{N} \sum_{n \sim N} \int_{0}^{1} \int_{\frac{1}{2}}^{1} d x d y\left|\sum_{m \sim M} e\left(\left(2 n x+4 \lambda n^{3} y\right) m+\left(x+6 \lambda n^{2} y\right) m^{2}+4 \lambda n y m^{3}+\lambda m^{4} y\right)\right|^{p}
$$

by

$$
\left.\frac{1}{N^{1-\varepsilon}} \sum_{n \sim N} \int_{0}^{1} \int_{0}^{1} \int_{\frac{1}{2}}^{1} d x^{\prime} d y^{\prime} d y\left(\sum_{m \sim M} e\left(x^{\prime} m+y^{\prime} m^{2}+4 \lambda n y m^{3}+\lambda m^{4} y\right)\right)\right|^{p}
$$

Since $\left[\frac{1}{2}, 1\right] \times\{n \sim N\} \rightarrow[0,4 N] \times[0,1]:(y, n) \mapsto(4 n y, y)$ induces a measure bounded by the uniform measure at scale $1 \times d w^{\prime}$, it follows that at scale $\frac{1}{M} \times \frac{1}{M^{2}} \times \frac{1}{\lambda M^{3}} \times \frac{1}{\lambda M^{4}} \mu$ may be majorized by uniform measure up to a factor $N^{\varepsilon}\left(1+\lambda M^{3}\right)$. Hence (5.13) may be bounded by

$$
N^{\varepsilon}\left(1+\lambda M^{3}\right) \int_{0}^{1} \int_{0}^{1} \int_{0}^{1} \int_{0}^{1}\left|\sum_{m \sim M} e\left(x^{\prime} m+y^{\prime} m^{2}+\lambda N z^{\prime} m^{3}+\lambda w^{\prime} m^{4}\right)\right|^{p} d x^{\prime} d y^{\prime} d z^{\prime} d w^{\prime} .
$$


One may do better. Assume $\lambda M^{3}>100$ and shift in (5.13) the $y$-variable by $o\left(\frac{1}{\lambda M^{4}}\right)$, i.e. replace $y$ by $y+\frac{z}{\lambda M^{4}}, z=o(1)$. One obtains

$\frac{1}{N^{1-\varepsilon}} \sum_{n \sim N} \int_{0}^{1} \int_{0}^{1} \int_{\frac{1}{2}}^{1} \int_{0}^{1} d x^{\prime} d y^{\prime} d y d z\left|\sum_{m \sim M} e\left(x^{\prime} m+y^{\prime} m^{2}+4 \lambda n\left(y+\frac{z}{\lambda M^{4}}\right) m^{3}+\lambda m^{4} y\right)\right|^{p}$.

Assuming

$$
\lambda M^{4}<N
$$

we note that for fixed $\frac{1}{2} \leq y \leq 1$, the map $(n, z) \mapsto n\left(y+\frac{z}{\lambda M^{4}}\right)$ induces a normalized measure essentially bounded by $\frac{1}{N} 1_{[0,2 N]}$. Consequently, under the condition (5.15), (5.13) is bounded by

$$
N^{\varepsilon} \int_{0}^{1} \int_{0}^{1} \int_{0}^{1} \int_{0}^{1} \mid \sum_{m \sim M} e\left(x^{\prime} m+y^{\prime} m^{2}+\lambda N z^{\prime} m^{3}+\left.\lambda w^{\prime} m^{4}\right|^{p} d x^{\prime} d y^{\prime} d z^{\prime} d w^{\prime} .\right.
$$

Taking $M<N^{\frac{2}{3}}$, (5.15) will hold for $\lambda<N^{-5 / 3}$.

\subsection{Application of mean value theorems.}

Use the 8th moment bound ([B-I2]), or equivalently, Theorem 2 in the paper, we get

$$
\max _{\left|c_{m}\right| \leq 1} \iiint d x^{\prime} d y^{\prime} d z^{\prime}\left|\sum_{m \sim M} c_{m} e\left(x^{\prime} m+y^{\prime} m^{2}+\frac{z^{\prime}}{M} m^{3}\right)\right|^{8} \ll M^{4+\varepsilon}
$$

Application of (5.17) to (5.16) with fixed $w^{\prime}$ and $p=8$ implies then

$$
\int_{0}^{1} \int_{\frac{1}{2}}^{1}\left|\sum_{n \sim N} e\left(n^{2} x+\lambda n^{4} y\right)\right|^{8} d x d y \ll N^{\varepsilon}\left(\frac{N}{M}\right)^{5} M^{4}\left(1+\frac{1}{\lambda N M^{2}}\right) \ll N^{4+\frac{1}{3}+\varepsilon}+\frac{N^{2+\varepsilon}}{\lambda}
$$

taking $M=N^{\frac{2}{3}-\varepsilon}$ and $N^{-3+\varepsilon}<\lambda<N^{-2}$.

Braking up the range $y \in[0,1]$ in sub-intervals $\left[\frac{1}{2} \sigma, \sigma\right], \sigma=2^{-s}$ a change of variables and replacement of $\lambda$ by $N^{-\frac{7}{3}} \sigma$ in (5.18) gives

$$
\int_{0}^{1} \int_{N^{-\frac{2}{3}}}^{1}\left|\sum_{n \sim N} e\left(n^{2} x+N^{-\frac{7}{3}} n^{4} y\right)\right|^{8} d x d y \ll N^{\frac{13}{3}+\varepsilon} .
$$

The remaining range is simply bounded by

$$
N^{-\frac{2}{3}} I_{8}\left(N^{-3}\right) \leq N^{\frac{4}{3}} I_{6}\left(N^{-3}\right) \ll N^{\frac{13}{3}+\varepsilon} .
$$

Hence we establish 0.17 . 


\section{Theorem 11.}

$$
I_{8} \leq I_{8}\left(N^{-\frac{7}{3}}\right) \ll N^{\frac{13}{3}+\varepsilon}
$$

Next, one may consider the 10th moment. Setting $p=10$ in (5.9) implies with $M=N^{\frac{2}{3}-\varepsilon}, N^{-\frac{8}{3}}<\lambda<N^{-\frac{5}{3}}$

$$
\begin{aligned}
& \int_{0}^{1} \int_{\frac{1}{2}}^{1}\left|\sum_{n \sim N} e\left(n^{2} x+\lambda n^{4} y\right)\right|^{10} d x d y \ll \\
& N^{\varepsilon}\left(\frac{N}{M}\right)^{7} \int_{0}^{1} \int_{0}^{1} \int_{0}^{1} \int_{0}^{1}\left|\sum_{m \sim M} e\left(m x+m^{2} y+\lambda N m^{3} z+\lambda m^{4} w\right)\right|^{10} d x d y d z d w .
\end{aligned}
$$

Apply Theorem 4 with $\varphi_{2}(t)=t^{3}, \varphi_{3}(t)=t^{4}$ and $\delta=\lambda^{-1} N^{-1} M^{-3}$, $\Delta=\lambda^{-1} M^{-4}$

This gives the bound

$$
\begin{aligned}
& N^{\varepsilon}\left(\frac{N}{M}\right)^{7}\left\{\delta \Delta^{3 / 4} M^{7}+(\delta+\Delta) M^{6}+M^{5}\right\} . \\
\ll & N^{\varepsilon}\left(N^{2} \lambda^{-7 / 4}+N^{\frac{11}{3}} \lambda^{-1}+N^{\frac{17}{3}}\right) \ll N^{4+\varepsilon} \lambda^{-1}
\end{aligned}
$$

for $\lambda$ as above.

Thus

$$
\int_{0}^{1} \int_{N^{-1}}^{1}\left|\sum_{n \sim N} e\left(n^{2} x+N^{-\frac{5}{3}} n^{4} y\right)\right| d x d y \ll N^{\frac{17}{3}+\varepsilon} .
$$

The remaining range may be captured using (5.19), i.e.

$$
\int_{0}^{1} \int_{0}^{N^{-2 / 3}}\left|\sum_{n \sim N} e\left(n^{2} x+N^{-\frac{5}{3}} n^{4} y\right)\right|^{10} d x d y \ll N^{-\frac{2}{3}} N^{2} I_{8}\left(N^{-\frac{7}{3}}\right) \ll N^{\frac{17}{3}+\varepsilon} .
$$

Hence we establish Theorem 11.

\section{REFERENCES}

[B-D] J. Bourgain, C. Demeter, The proof of the $l^{2}$-decoupling conjecture, arXiv: 1405335.

[B-D2] J. Bourgain, C. Demeter, $\ell^{p}$ decouplings for hypersurfaces with nonzero Gaussian curvature, in preparation.

[B-G] J. Bourgain, L. Guth, Bounds on oscillatory integral operators based on multilinear estimates, GAFA 21 (2011), no 6, 1239-1295.

[B-I1] E. Bombieri, H. Iwaniec, On the order of $\zeta\left(\frac{1}{2}+i t\right)$, Ann. Scuola Norm. Sup. Pisa Cl. Sci (4) 13 (1986), 449-472.

[B-I2] E. Bombieri, H. Iwaniec, Some mean value theorems for exponential sums, Ann. Scuola Norm. Sup.Pisa Cl. Sci (4) 13(1986), 473-486. 
[H] M.N. Huxley, Ares, Lattice Points and Exponential Sums, LMS monographs, 13 (1996).

[H4] M.N. Huxley, Exponential sums and the Riemann zeta function, IV, Proc. London Math. Soc. (3) 66 (1993), 1-40.

[H5] M.N. Huxley, Exponential sums and the Riemann zeta function, V, Proc. London Math. Soc (3) 90 (2005), 1-41,

[H-K] M.N. Huxley, G. Kolesnik, Exponential sums and the Riemann zeta function III, Proc. London Math. Soc. (3) 62 (1991), 449-468.

[P] S. Parsell, A note on Weyl's inequality for eight powers, (preprint)

[R-S] O. Robert, P.Sargos, Un théorème de moyenne pour les sommes d'exponentielles. Application à l'inégatite de Weil, Publ. Inst. math. (Beograd) N.S. 67 (2000), 14-30.

[W] T. Wooley, Mean value estimates for odd cubic Weyl sums, arXiv 1401.7152v1, (2014).

[W2] T. Wooley, Translation invariance, exponential sums and Waring's problem, arXiv:1404.3508 1 , (2014).

School of Mathematics, Institute for Advanced Study, 1 Einstein Drive, Princeton, NJ 08540 